\title{
Density Law of Spiral Galaxy Relative to Rotation Curves
}

\author{
Nazeeha A. Daoud
}

\author{
Aziz J. Abdul-Kahader \\ Department of Physics \\ College of Science \\ Mosul University
}

Mohammed K. Zeki

(Received 20 / 1 / 2009 ; Accepted 15 / 6 / 2009)

\begin{abstract}
An expression for galactic density has been derived from galactic rotation curves using Burbidge technique, in which rotational velocity $\mathrm{v}(\mathrm{r})$ is related to the galactic density at a given distance $r$. Abel's integral has been used to evaluate the galactic density $\rho_{(\mathrm{r}, \mathrm{k})}$. The derived density law has been applied to spiral galaxies with a general observed rotation curves with a turn-over at some distance $r_{t}$, so we got two different density forms $\rho_{1}(r, k)$ and $\rho_{2}(\mathrm{r}, \mathrm{k})$ for the core and for disk and halo of the galaxy respectively.
\end{abstract}

\section{المينة الريالنية لكألفةجرة حلزونية نسبة إلىمنحيلتسرعنها المورلنية}

\section{الملغ}

تضضمن البحث لمتخدلم ققنية بيريج في اشققاق صيغة رياضية للعلافة التي تربط كثلفة المجرة (r, k) وسرعة دورانها v(r,k) كدالة اللمسفة التطبية (r) من مركز المجرة.

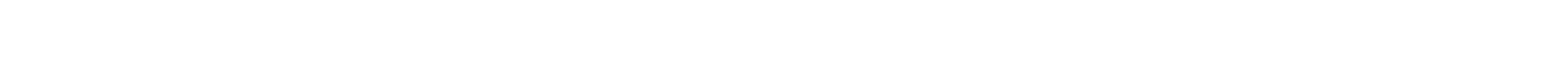

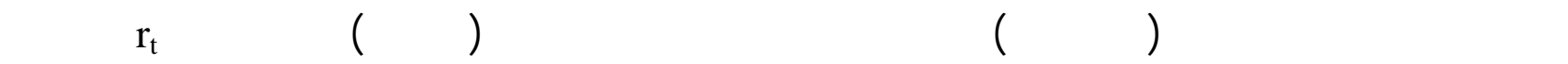

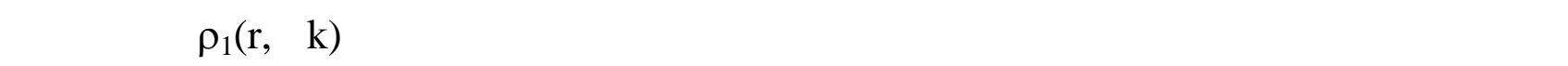

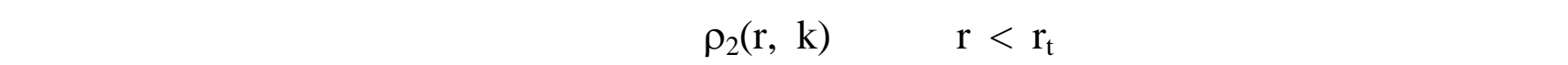

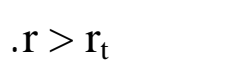




\section{INTRODUCTION}

Galaxies are disks of stars and gas which rotate around their center (center of the galaxy) due to the force of gravity holding them together. By measuring the radial speed at which stars and gas move, one can calculate galactic density.

Rotation curves are tools providing us informations about the distribution of mass, kinematic, evolution, and formation of galaxies (Sofu and Rubin, 2001).

Galactic rotation was discovered by Slipher (1914), when he detected inclined absorption lines in the nuclear spectra of M31. This led Pease (1918) to investigate the rotation of great nebula in Andromeda. After that rotation of galaxies was studied by many authors like Oort (1940), Mayall (1951), Burbidge and Burbidge (1960), Merritt et al., (1999) and Combes et al., (2000).

Rotation velocity (radial velocity) of galaxy can be measured from the spectrum of the galaxy by measuring the Doppler Shift of the emission lines (nutral hydrogen $21 \mathrm{~cm}$ line in the radio region, $\mathrm{H} \alpha$ in optical). From these observations one can construct the observed line of sight velocity of different parts of the galaxy.

The above observations can be used to construct a rotation curve of the galaxy (A plot of rotational velocity as a function of the radial distance from the center of the galaxy). There is one complication is that galaxies are typically inclined to our line of sight, so that, we are really measuring $\mathrm{v}(\mathrm{r}) \sin (\mathrm{i})$. In general it is straightforward to determine and correct for inclination.

In this work we use the technique of Burbidge, Burbidge, and Prendergast, (1959), Binney and Tremaine, (1987), we derived an expression for the density of spiral the galaxy. In this technique the rotational velocity $v(r)$ at a distance $r$ is related to the galactic density $\rho_{(\mathrm{r}, \mathrm{k})}$, via and integration over the radial distance from the center to $\mathrm{r}$.

Abel's integrals were used to solve our integral equation to get an expression for the galactic density.

Spiral galactic rotation curve has a turn over at some radius $r_{t}$, as shown in fig.(1), (Greisen et al., 2009) so we approximate the rotation curve by two simple relations for some radius less and greater than $r_{t}$, in this way we got two equations for the density law $\rho_{1(r, k)}$ for the inner part of the galaxy $\left(r<r_{t}\right)$, and $\rho_{2(r, k)}$ for the outer part of the galaxy $\left(r>r_{t}\right)$,

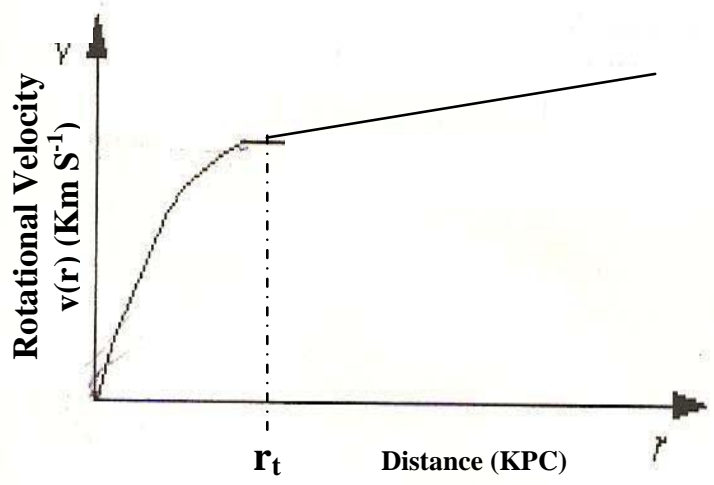

Fig. 1: A typical rotation curve of spiral galaxies taken from $21 \mathrm{~cm}$-line. 
The rotation curves of spiral galaxies differ from one galaxy to another as shown in fig.(2).

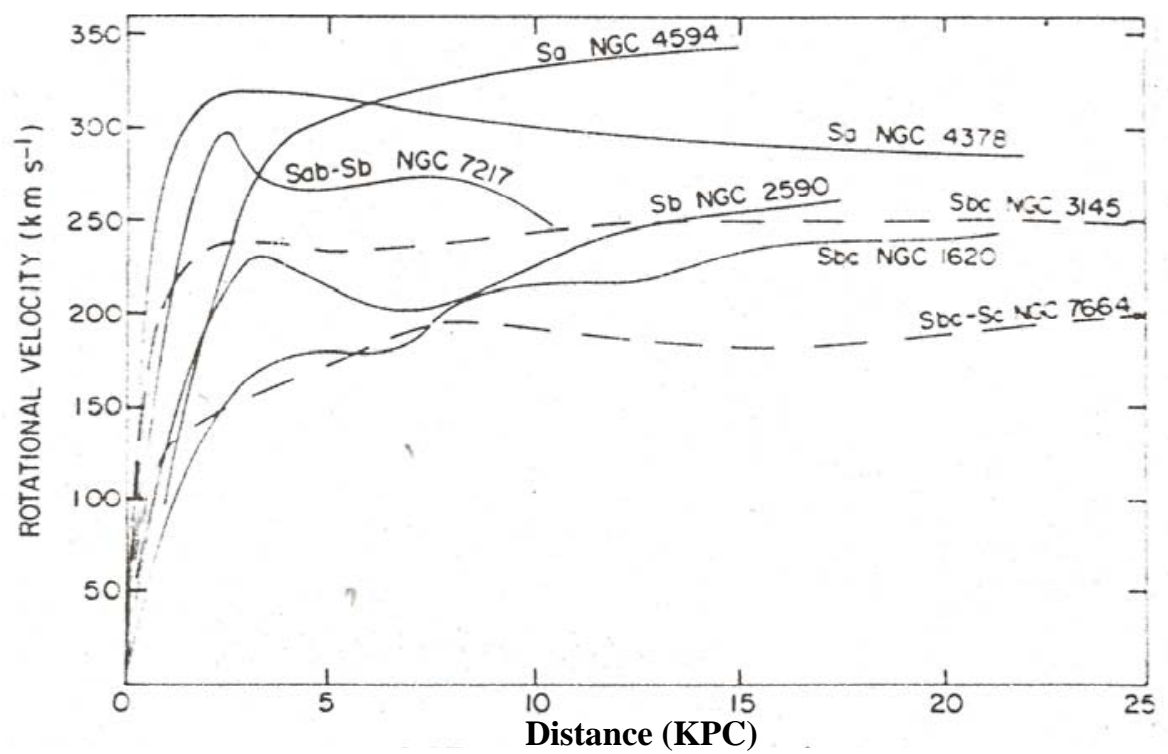

Fig. 2: A series of rotation curves for spiral galaxies (Figure from Rubin, Ford and Thonnard, AP. J. Lett. 255, L107, 1978.

Cited in Mihalas and Binney, 1968)

\section{CALCULATIONS AND RESULTS}

To derive the density relation of the galaxy, we start with Burbidge equation (Burbidge et al., 1959, Binney and Tremaine, 1987),

$$
\mathrm{v}^{2}(\mathrm{r}, \mathrm{k})=4 \pi \mathrm{G}\left(1-\mathrm{k}^{2}\right)^{1 / 2} \int_{0}^{\mathrm{r}} \frac{\rho(\mathrm{a}, \mathrm{k}) \mathrm{a}^{2}}{\left(\mathrm{r}^{2}-\mathrm{k}^{2} \mathrm{a}^{2}\right)^{1 / 2}} \mathrm{da}
$$

Where:

$\mathrm{v}(\mathrm{r}, \mathrm{k})$ : is the rotational velocity,

$\rho(\mathrm{a}, \mathrm{k})$ : is the galactic density at some distance $\mathrm{a}$,

$\mathrm{G}$ : is the gravitational constant.

a: is the semimajor axis,

$\mathrm{k}$ : is the eccentricity which depends on the semi axis length a and $\mathrm{b}\left(\mathrm{k}=\sqrt{1-\frac{\mathrm{b}^{2}}{\mathrm{a}^{2}}}\right), 0<\mathrm{k}<1$

The mass of a spheroidal shell :

$\operatorname{dm}(\mathrm{a}, \mathrm{k})=4 \pi \mathrm{a}^{2}\left(1-\mathrm{k}^{2}\right)^{1 / 2} \quad \rho(\mathrm{a}, \mathrm{k}) \mathrm{da}$

Then equation (1) becomes,

$\mathrm{v}^{2}(\mathrm{r}, \mathrm{k})=\mathrm{G} \int_{0}^{\mathrm{r}} \frac{\mathrm{dm}(\mathrm{a}, \mathrm{k})}{\left(\mathrm{r}^{2}-\mathrm{a}^{2} \mathrm{k}^{2}\right)^{1 / 2}}$

since surface density $\sigma(\mathrm{a}, \mathrm{k})$ is : 
$\sigma(\mathrm{a}, \mathrm{k})=\frac{\mathrm{dm}(\mathrm{a}, \mathrm{k})}{2 \pi \mathrm{a} \mathrm{da}}$

And, let

$\mathrm{t}=\frac{\mathrm{k}^{2} \mathrm{a}^{2}}{\mathrm{r}}$

Then equation (3) reads

$v^{2}(r, k)=\frac{G \pi r^{1 / 2}}{k^{2}} \int_{0}^{r} \frac{\sigma(t, k)}{(r-t)^{1 / 2}} d t$

or

$\frac{\mathrm{k}^{2}}{\pi \mathrm{G} \mathrm{r}^{1 / 2}} \mathrm{v}^{2}(\mathrm{r}, \mathrm{k})=\int_{0}^{\mathrm{r}} \frac{\sigma(\mathrm{t}, \mathrm{k})}{(\mathrm{r}-\mathrm{t})^{1 / 2}} \mathrm{dt}$

let

$\mathrm{f}_{(\mathrm{r}, \mathrm{k})}=\frac{\mathrm{k}^{2}}{\pi \mathrm{Gr} \mathrm{r}^{1 / 2}} \mathrm{v}^{2}(\mathrm{r}, \mathrm{k})$

then

$\mathrm{f}_{(\mathrm{r}, \mathrm{k})}=\int_{0}^{\mathrm{r}} \frac{\sigma(\mathrm{t}, \mathrm{k})}{(\mathrm{r}-\mathrm{t})^{1 / 2}} \mathrm{dt}$

Now comparing equation (7), with the following Abel's integral (Arfken, 1973),

$\mathrm{f}_{(\mathrm{x})}=\int_{\mathrm{o}}^{\mathrm{x}} \frac{\phi(\mathrm{t})}{(\mathrm{x}-\mathrm{t})^{1 / 2}} \mathrm{dt}$

$\phi(\mathrm{x})=\frac{1}{\pi} \frac{\mathrm{d}}{\mathrm{dx}} \int_{\mathrm{o}}^{\mathrm{x}} \frac{\mathrm{f}_{(\mathrm{t})}}{(\mathrm{x}-\mathrm{t})^{1 / 2}} \mathrm{dt}$

Then the surface galactic density equation becomes

$\sigma(\mathrm{r}, \mathrm{k})=\frac{2 \mathrm{k}^{4}}{\pi \mathrm{G}} \frac{\mathrm{d}}{\mathrm{dr}} \frac{1}{\mathrm{r}} \int_{\mathrm{o}}^{\mathrm{r}} \frac{\mathrm{v}^{2}(\mathrm{a}, \mathrm{k}) \mathrm{a}}{\left(\mathrm{r}^{2}-\mathrm{k}^{2} \mathrm{a}^{2}\right)^{1 / 2}} \mathrm{da}$

Now combining equations 2 , 4 , with equation 9 , we get,

$\rho(r, k)=\frac{k^{4}}{\pi^{2} G\left(1-k^{2}\right)^{1 / 2} r} \frac{d}{d r}\left[\frac{1}{r} \int_{0}^{r} \frac{V^{2}(a, k) a}{\left(r^{2}-k^{2} a^{2}\right)^{1 / 2}} d a\right]$

Equation (10) is a general galactic density law as a function of rotational velocity.

Observed curves for spiral galaxy derived from $21 \mathrm{~cm}$ line fig. (1), show a turn over at some radius $r_{t}$, in this case the rotational curves can be approximated by two equations of line, i.e.

$\mathrm{v}(\mathrm{a})=\alpha \mathrm{a} \quad$ at $\quad \mathrm{a}<\mathrm{r}_{\mathrm{t}}$

and,

$\mathrm{v}(\mathrm{a})=\beta \mathrm{a}+\gamma \quad$ at $\quad \mathrm{a}>\mathrm{r}_{\mathrm{t}} \quad \beta<<1$

Where $\alpha, \beta$, and $\gamma$ are constants taken from the observed rotation curves, which differ from galaxy to another.

let $\quad \rho_{1}(\mathrm{r}, \mathrm{k}) \quad \mathrm{be}$ the density for $\quad \mathrm{v}(\mathrm{a})=\alpha \mathrm{a}$,

and, $\rho_{2}(r, k) \quad$ be the density for $\quad \mathrm{v}(\mathrm{a})=\beta \mathrm{a}+\gamma$, 
then by substituting equation (11a) in equation (10), and taking $x=\frac{r}{k}$, we get,

$\rho_{1(\mathrm{r}, \mathrm{k})}=\frac{\mathrm{k}^{3} \alpha^{2}}{\mathrm{G} \pi^{2}\left(1-\mathrm{k}^{2}\right)^{1 / 2} \mathrm{r}} \frac{\mathrm{d}}{\mathrm{dr}}\left[\frac{1}{\mathrm{r}} \int_{0}^{\mathrm{r}_{\mathrm{t}}} \frac{\mathrm{a}^{3}}{\left(\mathrm{x}^{2}-\mathrm{a}^{2}\right)^{1 / 2}} \mathrm{da}\right]$

the integral in equation (12) is evaluated using standard integral (William, 1985), so equation (12) becomes.

$$
\begin{aligned}
& \rho_{1}(\mathrm{r}, \mathrm{k})=\frac{\alpha^{2}}{3 G \pi^{2} \sqrt{1-\mathrm{k}^{2}}}\left[4-4 \sqrt{1-\mathrm{k}^{2} \frac{\mathrm{r}_{\mathrm{t}}^{2}}{\mathrm{r}^{2}}}-\frac{\mathrm{k}^{4} \frac{\mathrm{r}_{\mathrm{t}}^{4}}{\mathrm{r}^{4}}+2 \mathrm{k}^{2} \frac{\mathrm{r}_{\mathrm{t}}^{2}}{\mathrm{r}^{2}}}{\sqrt{1-\mathrm{k}^{2} \frac{\mathrm{r}_{\mathrm{t}}^{2}}{\mathrm{r}^{2}}}}\right] \\
& \mathrm{r}<\mathrm{r}_{\mathrm{t}} \\
& 0<\mathrm{k}<1
\end{aligned}
$$

Which represent the galactic density of the spiral galaxy in the region $\mathrm{r}<\mathrm{r}_{\mathrm{t}}$.

In the same way combining (11b) and (10) we get,

$$
\rho_{2}(r, k)=\frac{k^{3}}{G \pi^{2}\left(1-k^{2}\right)^{1 / 2} r} \frac{d}{d r}\left[\frac{1}{r} \int_{r_{t}}^{r} \frac{\left(\beta^{2} a^{2}+2 \beta \gamma a+\gamma^{2}\right) a}{\left(x^{2}-a^{2}\right)^{1 / 2}} d a\right]
$$

Evaluating the three integrals in the above equation, using the standard integrals, we get

$$
\begin{aligned}
& \rho_{2}(r, k)=\frac{\beta^{2}}{3 G \pi^{2} \sqrt{1-k^{2}}}\left[2 \sqrt{k^{2}-1}\left(k^{2}+2\right)+4 \sqrt{1-k^{2} \frac{r_{t}^{2}}{r^{2}}}+k^{2} \frac{r_{t}^{2}}{r^{2}} \frac{\left(k^{2} \frac{r_{t}^{2}}{r^{2}}+2\right)}{\sqrt{1-k^{2} \frac{r_{t}^{2}}{r^{2}}}}\right] \\
& +\frac{\beta \gamma k^{2}}{G \pi^{2} \sqrt{1-k^{2}}}\left[\frac{\operatorname{Sin}^{-1}(k)-k \sqrt{1-k^{2}}}{k}+\frac{k^{2} \frac{r_{t}^{3}}{r^{3}}}{\sqrt{1-k^{2} \frac{r_{t}^{2}}{r^{2}}}}+\frac{\operatorname{Sin}^{-1}\left(k \frac{r_{t}}{r}\right)}{k}+\frac{\frac{r_{t}}{r}}{\sqrt{1-k^{2} \frac{r_{t}^{2}}{r^{2}}}}\right] \frac{1}{r} \\
& +\frac{\gamma^{2} \mathrm{k}^{4}}{\mathrm{G} \pi^{2} \sqrt{1-\mathrm{k}^{2}}}\left(\frac{\frac{\mathrm{r}_{\mathrm{t}}^{2}}{\mathrm{r}^{2}}}{\sqrt{1-\mathrm{k}^{2} \frac{\mathrm{r}_{\mathrm{t}}^{2}}{\mathrm{r}^{2}}}}\right) \frac{1}{\mathrm{r}^{2}} \quad \mathrm{r}>\mathrm{r}_{\mathrm{t}}, 0<\mathrm{k}<1
\end{aligned}
$$

Equation (15) represent the galactic density of the spiral galaxy in the region $r>r_{t}$.

\section{DISCUSSION}

Galactic rotation depends on the distribution of their mass, so a great deal can be learned about the matter in galaxies by studying galactic rotation curves. Rotational curves do not follow the expected inverse square root law, but the curves are flat (rather than 
dropping down outside the center bulge of the galaxy), the speed is nearly a constant function of radius as shown in fig. (1).

Equation (10) is a general density law for all galaxies, the density is a function of eccentricity $\mathrm{k}$ of the galaxy, and it's rotational velocity $\mathrm{v}(\mathrm{r})$. The calculated density of spiral galaxies differ from one galaxy to another, due to the slightly difference in their rotational curves fig. (2).

Equation (13) shows that $\left(\frac{r_{t}}{r}\right)$ is of the same order (dimensionless), therefore $\rho_{1(r, k)}$ is almost $\mathrm{r}$ independent, so it depends upon the $\mathrm{k}$ and $\alpha$ only, where $\alpha$ can be taken from the observed rotation curves of a given galaxy. This result for $\rho_{1(\mathrm{r}, \mathrm{k})}$ is in agreement with most text book in which the core density is constant (Binney and Tremaine, 1987).

Equation (15) shows that the density $\rho_{2(r, k)}$ of the outer parts (disk an halo) consist of three terms, the first one is almost constant for $\left(\frac{r_{t}}{r}\right)$ is of the same order. For the same reasons the second and the third terms are of order $\mathrm{r}^{-1}$ and $\mathrm{r}^{-2}$ respectively, beside that $\rho_{2(\mathrm{r}, \mathrm{k})}$ depends upon the encentricity $k$, and the constants $\beta, \gamma$ which can be taken from the observed rotation curves.

Rotational curves have been a very efficient tool to study the most distribution in spiral galaxies. The most clear evidence of the existence of dark matter in spiral galaxies comes from their rotational velocities.

Constructed rotational curve of the halo depending on the galactic luminosity (Ouellettem Amram and Carignam 2001; Yegorova, 2007 and Sofue et al., 2008) can be used in future to estimate the halo mass and the amount of dark matter in the halo.

\section{REFERENCES}

Arfken, G. (1973). "Mathematical Methods for Physicist". 2nd. edn., Academic Press. 734p. Binney, J.; Tremaine, S. (1987). "Galactic Dynamics". Princeton NJ: Princeton Univ., Press. 58p.

Burbidge, E. M.; Burbidge, G.R. (1960). Distortion of the plane of the oscculting matter in NGC 5866. AP. J. ,131, 224-226.

Burbidge, E. M.; Burbidge, G.R.; Prendergast, K. (1959). Mass distribution and physical condition in the inner region of NGC 1068. AP. J. 130, 739.

Combes, F.; Mamon, G.A.; Charmandaris, V. Eds. (2000). "Dynamics of Galaxies: From the Early Universe to the Present, XVth IAP Mech". ASP Conf., Ser., Vol. 197. San Francisco, Astron Soc., Pac.

Greisen, E. W.; Spekkens, K.; Moorse, G. A.(2009). Aperture synthesis observations of the nearby spiral NGC 6503. Modeling the Thin and Thick H1 Disks. The Astron. J. 137, 4718-4733.

Mayall, Nu. (1951)." In the Structure of Galaxy. Ann. Arbor": Univ. Mich. Press, 19p.

Merritt, D.; Sellwood, J.A.; Valluri, M., Eds. (1999). "Galaxy Dynamics". ASP. Conf., Soc. Vol. 182, San Francisco, Astron Soc., Pac.

Mihalas, D. ; Binney, J. (1968). "Galactic Astronomy, Structure and Kinematics". W. H. Freeman an Company. 958p. 
Ooret, J.H. (1940). Some problems concering the structure and dynamics of the galactic system and elliptical nebulae NGC 3115 and 4494, AP. J. 91, 273.

Ouellett, S. R.; Amram, P. ; Carignan, C. (2001). Accurate determination of the mass distribution IN spiral galaxies. II. Testing the shape of dark halo. The Astron. J., 121, 1952-1964.

Pease, F.G. (1918). Proc. Natl. Acad. Sci. USA 4:21. Cited in Soufu Y. and Rubin V. 2001.

Rubin, V. C.; Ford, W. K. Jr.; Thonnard, N. (1978). AP. J. Lett, 255, L107. Cited in Mihalas, D. and Binney, J. 1968.

Slipher, V. M. (1914). Lowell Obs. Bull. 62, Vol. 11, 12. Cited in Sofu, Y. and Rubin V. 2001.

Sofu, Y. ; Rubin, (2001). Rotation Curves of Spiral Galaxy. Annu. Rev. Astron. 39, 137-74

Sofue, Y.; Honma, M. ; Omodaka, T. (2008). "Unified Rotation Curve of the GalaxyDecomposition into de Vaucouleurs Bulge, Disk, Dark Halo, and the 9-Kpc Rotation Dip-Astronomical Society of Japan". pp. 1-11.

William, H.; Beyer, (1985). "Standard Mathematical Tables". $7^{\text {th }}$ edn., CRC Press Inc. pp. 253-254.

Yegorova, I. A. (2007). Dark Matter in Spiral Galaxies. PH. D. Thesis. Scuola International Superiore DI Studi Avanzati. International School for Advanced Studies. pp. 1-137. 